\title{
Mortalidade por tumores de cérebro no Brasil, 1980-1998
}

\author{
Brain tumors mortality in Brazil, 1980-1998
}

Gina Torres Rego Monteiro 1

Sergio Koifman 1

\footnotetext{
1 Departamento de Epidemiologia e Métodos Quantitativos em Saúde, Escola Nacional de Saúde Pública, Fundação Oswaldo Cruz. Rua Leopoldo Bulhões 1480, Rio de Janeiro, $R J$ 21041-210, Brasil. gtorres@cremerj.com.br koifman@ensp.fiocruz.br
}

\begin{abstract}
Brain tumors are rare, but their incidence and mortality have increased in different countries, including Brazil, especially among the elderly. This paper presents the mortality pattern of brain tumors in Brazil, including distribution of mortality rates by gender, age, topography, and tumor staging, from 1980 to 1998. Age-adjusted (by world population) brain cancer mortality rates increased from 2.24/100,000 to 3.35/100,000 inhabitants among the overall population during this period (an increase of 50\%). Observed rates were higher during childhood than adolescence and increased with age, reaching the highest levels among the elderly. Among individuals 70 years of age and older, an average annual increase of 6\% was observed in the series. Brain and meningeal tumor rates are presented for the entire country and selected State capitals. Further study is needed to elucidate the role of both new diagnostic technologies and environmental exposures potentially associated with the observed changes in brain cancer mortality rates.

Key words Central Nervous System; Brain Neoplasms; Neoplasms; Mortality

Resumo Os tumores cerebrais são raros, mas sua incidência e mortalidade vêm aumentando ao longo das últimas décadas em vários países, inclusive no Brasil, particularmente entre idosos. $O$ presente artigo descreve o perfil da mortalidade desses tumores no país, analisando a distribuição de suas taxas segundo sexo, idade, topografia e natureza tumoral entre 1980 e 1998. As taxas de mortalidade por esta neoplasia, ajustadas por idade pela população mundial, cresceram de 2,24/100 mil para 3,35/100 mil, correspondendo a um aumento de 50\%. Estas foram mais elevadas na infância que na adolescência, aumentando posteriormente com a idade e alcançando níveis altos nos mais idosos, sendo o crescimento médio nos maiores de 70 anos de $6 \%$ ao ano ao longo da série estudada. São analisadas as taxas de tumores do encéfalo e das meninges cerebrais para o país e para capitais selecionadas. A constatação do aumento dessas taxas demanda a elaboração de estudos com vistas a elucidar o papel de novas tecnologias diagnósticas, assim como de potenciais fatores de risco ambientais.
\end{abstract}

Palavras-chave Sistema Nervoso Central; Neoplasias Cerebrais; Câncer; Mortalidade 


\section{Introdução}

Os tumores de cérebro são raros, mas sua incidência e mortalidade vêm aumentando ao longo das últimas décadas em vários países, sobretudo entre idosos (Davis et al., 1990; Greig et al., 1990; Modan et al., 1992). Esse fato tem motivado a busca de explicações, gerando um debate em relação a tratar-se, ou não, de aumento real dessas neoplasias.

A constatação do incremento das taxas de tumores de cérebro despertou o interesse de pesquisadores, particularmente de países desenvolvidos, sendo publicados diversos artigos sobre a evolução de sua distribuição (Bahemuka et al., 1988; Fleury et al., 1997; Garfinkel \& Sarokhan, 1982; Legler et al., 1999). Esses trabalhos analisaram dados de mortalidade e/ ou incidência de tumores cerebrais referentes a diversos períodos entre 1940 e 1996, sendo também avaliada a possível contribuição, ao longo do tempo, dos procedimentos utilizados para diagnóstico desses tumores (Desmeules et al., 1992; Greenwald et al., 1981; Helseth, 1995).

Alguns autores (Greenwald et al., 1981; Helseth, 1995; Legler et al., 1999; Mao et al., 1991; Modan et al., 1992; Preston-Martin, 1996; Werner et al., 1995), consideraram a tendência ascendente das taxas de incidência de tumores de cérebro um artefato relacionado com a introdução de técnicas diagnósticas mais precisas e menos invasivas (tomografia computadorizada de crânio no início da década de 70 e ressonância magnética, no final da mesma década), a ampliação do acesso à atenção médica para as várias camadas da população, a modificação na composição etária populacional levando a um aumento da população idosa sob risco, a mudança de atitude em relação ao cuidado com os idosos (inclusive quanto a diagnóstico e tratamento) e as mudanças na classificação internacional de doenças (CID).

Contudo, outros autores (Ahlbom, 1990; Bahemuka et al., 1988; Greig et al., 1990), demonstraram que, embora essas mudanças tenham tido um efeito real sobre a incidência, há evidências de que sejam apenas parcialmente responsáveis pelo incremento observado, principalmente em relação aos novos procedimentos diagnósticos, uma vez que esse aumento já vinha ocorrendo antes da sua introdução. Alguns autores (Ahlbom, 1990; Fleury et al., 1997; Gold, 1982; Shugg et al., 1994), relatam que ocorreu um aumento real nessas taxas e sugerem que não podem ser desconsideradas hipóteses etiológicas relacionadas com o ambiente, como a possível introdução de carcinógeno com longo período de latência ou a redução da exposição a algum fator de proteção. Assim, recomendam a realização de estudos que avaliem eventos que possam estar relacionados ao aparecimento de tumores de cérebro.

Embora se conheça relativamente pouco sobre a etiologia dos tumores cerebrais, acredita-se que fatores genéticos, hormonais e ambientais estejam envolvidos (Bohnen \& Kurland, 1995; Gold, 1982). Condições relacionadas com os antecedentes patológicos pessoais como traumatismo craniano, epilepsia, viroses e outras infecções, padrão de dieta, contato com animais, exposição domiciliar a pesticidas e exposição à radiação eletromagnética (ionizante e não ionizante), têm sido associadas ao aumento do risco para câncer de cérebro (Gold, 1982; Inskip et al., 1995; Preston-Martin, 1996). A exposição ocupacional a derivados do petróleo, a produção de borracha e agroquímicos também tem sido relacionada a um maior risco de desenvolvimento da doença (Brem et al., 1995; Inskip et al., 1995; Ryan et al., 1992; Thomas \& Waxweiler, 1986).

Em consonância com o observado em outros países do mundo, os registros de câncer de base populacional no Brasil revelam um aumento das taxas dos tumores malignos de cérebro, especialmente na população idosa. Em 1987, em Porto Alegre, o coeficiente de incidência de câncer de cérebro e sistema nervoso, em indivíduos do sexo masculino com mais de 65 anos de idade, alcançou 120 casos novos por 100 mil habitantes, configurando uma variação três vezes maior que as taxas observadas no período 1978-1982 (Koifman, 1995).

O objetivo do presente artigo é descrever o perfil da mortalidade por tumores de cérebro no país, buscando assim caracterizar as tendências de sua distribuição nas décadas de 80 e 90.

\section{Material e métodos}

A denominação tumores do cérebro empregada neste texto compreende as neoplasias do encéfalo, das meninges cerebrais e dos nervos intracranianos, quer sejam benignas, malignas ou de comportamento incerto/desconhecido, conforme classificação proposta por PrestonMartin (1996).

Os dados de mortalidade foram obtidos do Sistema de Informação sobre Mortalidade (SIM), que consolida os óbitos ocorridos no país (DATASUS, 2000). A propriedade do uso desse sistema fundamenta-se em sua abrangência nacional, na utilização de regras universais para o preenchimento e a codificação das declarações 
de óbito que dão origem à base de dados de mortalidade, na observação criteriosa do processo de codificação das variáveis da declaração por profissionais treinados e no controle de qualidade a que é submetido regularmente. A qualidade da causa básica tem sido avaliada em algumas regiões do Brasil, revelando confiabilidade e validade adequadas para estudos epidemiológicos em neoplasias (Monteiro et al., 1997; Paula et al., 1994; Siqueira et al., 1999).

O SIM utilizou a 9a Revisão da CID (CID-9) para codificar os óbitos ocorridos de 1980 a 1995 e a 10a Revisão (CID-10) a partir de 1996.

Na CID-9 (OMS, 1985), os tumores do Sistema Nervoso estão agrupados nas seguintes categorias: 191 (Neoplasma maligno de encéfalo), 192 (Neoplasma maligno de outras partes e de partes não especificadas do sistema nervoso), 225 (Neoplasma benigno do encéfalo e de outras partes do sistema nervoso), 237 (Neoplasma de comportamento incerto das glândulas endócrinas e do sistema nervoso) e 239 (Neoplasma de natureza não especificada).

Na CID-10 (OMS, 1997), os tumores do Sistema Nervoso são discriminados nas categorias: C70 (Neoplasia maligna das meninges), C71 (Neoplasia maligna do encéfalo), C72 (Neoplasia maligna da medula espinhal, dos nervos cranianos e de outras partes do sistema nervoso central), D32 (Neoplasia benigna das meninges), D33 (Neoplasia benigna do encéfalo e de outras partes do sistema nervoso central), D42 (Neoplasia de comportamento incerto ou desconhecido das meninges) e D43 (Neoplasia de comportamento incerto ou desconhecido do encéfalo e do sistema nervoso central).

A correspondência entre as duas classificações, no que tange aos tumores cerebrais, pode ser constatada na Tabela 1.

A análise da série de meningiomas deve levar em conta as mudanças na classificação ocorridas com a introdução da CID-10, em 1996. A CID-9 contava com código próprio para as neoplasias de meninges encefálicas malignas (191.2) e benignas (225.2), assim como para as meninges espinhais malignas (192.3) e benignas (225.4). O óbito por tumor maligno da meninge poderia ser classificado como 192.8, "outros”, se a Declaração de Óbito (DO) não informasse claramente seu local de origem, mas registrasse o comprometimento de dois ou mais sítios listados na categoria 192 "neoplasma maligno de outras partes e de partes não especificadas do sistema nervoso", segundo a nota no 4 para classificação das neoplasias (OMS, 1985). Quando a DO descrevia a lesão da meninge como de comportamento incerto ou de natureza não especificada, ela deveria ser clas- sificada como 239.7 "neoplasmas de natureza não especificada das glândulas endócrinas, $e$ de outras partes do sistema nervoso". Já a CID10 apresenta códigos próprios para os meningiomas encefálicos malignos (C70.0) e benignos (D32.0), e para os tumores das meninges espinhais malignos (C70.1) e benignos (D32.1). Discrimina, ainda, subcategorias para os tumores malignos (C70.9) e benignos (D32.9) das meninges em que a DO não especificou se eram encefálicos ou espinhais. Os tumores de comportamento incerto ou desconhecido também contam com códigos específicos: encefálicos (D42.0), espinhais (D42.1) e meninge não especificada (D42.9).

Neste trabalho foi avaliada a distribuição de freqüências de tumores cerebrais em relação ao total de óbitos por neoplasias, localização anatômica (encéfalo, meninges e nervos cerebrais) e natureza tumoral (neoplasia maligna versus benigna), no período de 1980 a 1998. Foi analisada sua taxa de mortalidade global, por faixa etária e por sexo, e a variação das taxas ajustadas por idade ao longo do período. Levando-se em conta o número relativamente reduzido de óbitos, as análises foram realizadas por meio da determinação de taxas médias bienais, com objetivo de suavizar as tendências.

A distribuição etária da mortalidade foi analisada inicialmente por estratos de cinco anos, nos menores de 20 anos, e extratos de dez anos para os maiores. Realizou-se uma análise mais detalhada dos adultos, com o objetivo de avaliar possíveis diferenças em sua evolução segundo faixas etárias mais amplas, e determinar sua variação anual no período.

Para a análise da variação anual dos indicadores, foram determinadas as taxas de mortalidade padronizadas pela população mundial (Segi, 1960) e sua variação no período, sendo esta calculada por meio da expressão (Coleman et al., 1993) abaixo:

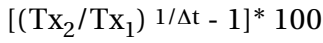

Onde: $\mathrm{Tx}_{1}=$ taxa de mortalidade no início do período; $\mathrm{Tx}_{2}=$ taxa de mortalidade no final do período; e $\Delta \mathrm{t}=$ intervalo de tempo entre as taxas comparadas.

Os óbitos por tumores do encéfalo foram examinados segundo as mesmas variáveis: natureza tumoral, idade, sexo e variação das taxas ajustadas por idade entre 1980 e 1997 . A análise por área geográfica abarcou o país e capitais selecionadas, sendo calculadas as taxas médias bienais, quando referentes ao país, e trienais para as capitais.

A escolha das cidades foi realizada levando-se em conta as maiores taxas brutas em 
Tabela 1

Correspondência entre a CID-9 e CID-10, no que tange aos tumores cerebrais.

\begin{tabular}{llll}
\hline Sítio & Natureza tumoral & CID-9 & CID-10 \\
\hline Encéfalo & Maligno & 191 & C71 \\
& Benigno & 225.0 & D33.0, D33.1, D33.2 \\
& Comportamento incerto1 & 237.52 & D43.0, D43.1, D43.2 \\
& Natureza não especificada1 & 239.6 & C70.0 \\
Meninges encefálicas & Maligno & 192.1 & D32.0 \\
& Benigno & 225.2 & D42.0 \\
Comportamento incerto1 & 237.63 & 239.74 & C72.2, C72.3, C72.4, \\
Nutras partes do Sistema & Natureza não especificada1 & $192.0,192.9$ & C72.5, C72.8, C72.9 \\
Nervoso Central & Maligno & & D33.3, D33.7, D33.9 \\
& Benigno & $225.1,225.9$ & D43.3, D43.7, D43.9
\end{tabular}

1 Tumores que não podem ser classificados como malignos nem como benignos são encontrados na CID-9 em duas subdivisões ("comportamento incerto" e "natureza não especificada"; na CID-10 são agrupados como neoplasias de "comportamento incerto ou desconhecido".

2 A categoria 237.5 inclui neoplasma de comportamento incerto de encéfalo e da medula espinhal não sendo possível separá-los.

3 A categoria 237.6 inclui neoplasma de comportamento incerto das meninges encefálicas e espinhais, não sendo possível separá-los.

4 A categoria 239.7 inclui neoplasmas de natureza não especificada das glândulas endócrinas e de outras partes do sistema nervoso, não sendo possível separá-los.

quatro momentos: 1980, 1985, 1990 e 1995. Assim, observando as dez capitais com taxas mais elevadas para tumores encefálicos em cada um desses anos, foram selecionadas: Belo Horizonte, Curitiba, Porto Alegre, Rio de Janeiro e São Paulo por estarem presentes nos quatro anos analisados; Fortaleza, Goiânia e João Pessoa, em dois e Belém, que só constava na relação de taxas elevadas em 1990, para incluir uma capital da Região Norte.

Os óbitos por tumores das meninges cerebrais também foram avaliados quanto à natureza tumoral, sexo, idade e variação das taxas ajustadas por idade, para o país e capitais selecionadas. Levando-se em consideração a mudança na classificação e o número ainda menor de óbitos, foram analisados em intervalos de tempo quadrienais de 1980 a 1995, tanto para o país quanto para as capitais, acrescidos de análise específica do triênio 1996/1998. As capitais foram escolhidas em processo similar ao executado nos tumores encefálicos, tendo sido selecionadas Belém, Belo Horizonte, Brasília, Rio de Janeiro e São Paulo.

\section{Resultados}

\section{Tumores do cérebro}

Os óbitos por câncer de cérebro corresponderam a 3,4\% do total de mortes por câncer em 1980 (2.098 em 61.253 óbitos) e a 4,4\% em 1998 (4.960 em 110.765).

No que se refere à localização anatômica no período de 1980 a 1998, mais de $90 \%$ estavam situados no encéfalo, entre 2,0 e $3,4 \%$ tiveram origem nas meninges, e menos de $1 \%$ em nervos cerebrais. Os tumores de partes não especificadas do cérebro corresponderam de 1,0 a $5,2 \%$ no período, repetindo-se esse perfil nas diversas faixas etárias.

A distribuição dos óbitos por tumores de cérebro quanto à sua natureza (neoplasia maligna ou benigna) apresentou grande instabilidade no período estudado. Embora os tumores malignos tenham sido quase sempre mais freqüentes, passaram a representar mais de $80 \%$ após a implantação da CID-10. Os tumores benignos foram pouco freqüentes, variando entre 2,1 e $5,9 \%$, enquanto os classificados como de natureza não especificada chegaram a representar mais da metade dos tumores cerebrais 
na CID-9, tendo se reduzido para $3,3 \%$ quando a CID-10 entrou em vigor.

A taxa bruta de mortalidade por tumores do cérebro apresentou um aumento de $59,0 \%$ ao longo do período: de 1,78/100 mil (1980/1981) para 2,83/100 mil (1996/1997). Esse incremento foi mantido mesmo após a padronização por idade, sendo da ordem de 2,24/ 100 mil no primeiro e 3,35/ 100 mil habitantes no último biênio (aumento de 49,9\%).

O crescimento médio foi de $2,8 \%$ ao ano, apresentando ampla variação nas diferentes faixas etárias, especialmente ao se analisar em separado as décadas de 80 e 90 (Figura 1). Os coeficientes de mortalidade nos grupos etários menores de 50 anos apresentaram declínio na década de 80 , mas cresceram no período seguinte. Já as taxas de mortalidade dos maiores de 50 apresentaram crescimento anual importante em toda a série, sendo que o extrato de 70 e mais anos cresceu aproximadamente $6 \%$ ao ano nos dois períodos.

O comportamento das taxas de mortalidade por tumores de cérebro por sexo mostrou perfil de crescimento semelhante, sendo aquelas referentes às mulheres sempre inferiores às observadas em homens. As taxas padronizadas por idade aumentaram de 1,92 para 3,00 óbitos por 100 mil mulheres $(56,2 \%)$ e de 2,57 para 3,75 óbitos por 100 mil homens $(45,9 \%)$. A razão de sexos esteve em torno de 1,3 nos biênios analisados.

As taxas de mortalidade por tumores cerebrais por faixa etária acompanharam o perfil descrito pela literatura, com taxas mais elevadas na infância que na adolescência, seguido de aumento com a idade chegando a níveis muito elevados nos mais idosos: a dos maiores de 70 anos quase triplicou no período analisado (Figura 2). Vale notar que no início da década de 80 a taxa de mortalidade desse grupo etário era menor do que a do grupo de 50 a 69 anos, mas ultrapassou-a ainda nesta década a partir do biênio 1984/1985 (dados não apresentados).

\section{Tumores do encéfalo}

Os óbitos por tumores do encéfalo representaram mais de $90 \%$ dos tumores cerebrais em todos os anos do período analisado, correspondendo a $95,4 \%$ em 1980, (2.002 em 2.098) e a 91,8\% em 1998 (4.469 em 4.869).

A distribuição quanto à natureza tumoral apresentou grande variabilidade no período. Sob a vigência da CID-9, os tumores malignos representaram de 43,2 a $73,2 \%$ dos tumores encefálicos, enquanto os de natureza não especi-
Figura 1

Variação anual média das taxas de mortalidade por tumores de cérebro, segundo faixa etária. Brasil, 1980-1997.

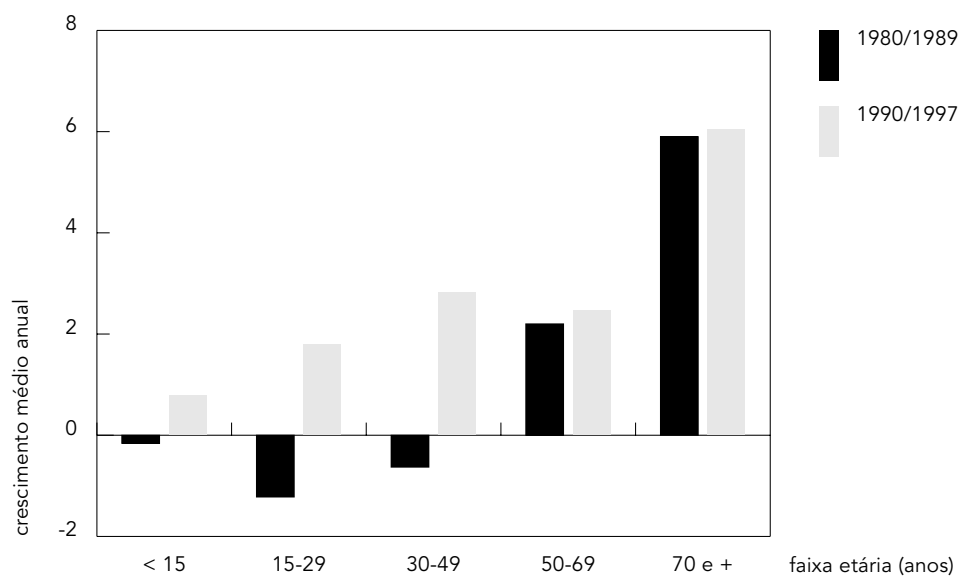

ficada responderam por 26,3 e $54,8 \%$. Os tumores benignos constituíram uma parcela reduzida dos tumores encefálicos, variando de 0,3 a 2,0\%. No biênio 1996/1997, cuja codificação foi pautada pela CID-10, os tumores malignos constituíram 87,9\% dos óbitos, os de comportamento incerto ou desconhecido $9,6 \%$ e os benignos, $2,5 \%$.

As taxas de mortalidade ajustadas por idade dos tumores do encéfalo apresentaram um crescimento constante nos 18 anos analisados, aumentando de 2,13 para 3,08/100 mil. A magnitude foi maior no sexo masculino todo o período, embora seu crescimento tenha sido maior nas mulheres. Enquanto as taxas dos homens aumentaram de 2,47 para $3,48 / 100 \mathrm{mil}$ $(40,7 \%)$, observou-se no sexo feminino uma elevação de 1,80 para $2,71 / 100$ mil (50,8\%). Esse comportamento se faz notar na razão de sexos que se manteve em 1,4 na década de 80 , passando para 1,3 nos biênios seguintes.

Quanto à distribuição por idade, os tumores encefálicos apresentaram taxas reduzidas entre os mais jovens, não ultrapassando 1 óbito por 100 mil habitantes nas faixas etárias menores de 30 anos, em nenhum biênio estudado. Os demais grupos etários apresentaram taxas crescentes com a idade e com o tempo. $\mathrm{O}$ crescimento mais importante, no entanto, foi observado nos maiores de 70 anos, cuja taxa em 1980/1981 era de 5,09/100 mil, chegando a 13,95/100 mil em 1996/97. Dessa maneira, ob- 
Figura 2

Taxas de mortalidade por tumores de cérebro, segundo faixa etária. Brasil, 1980/1981, 1988/1989 e $1996 / 1997$.

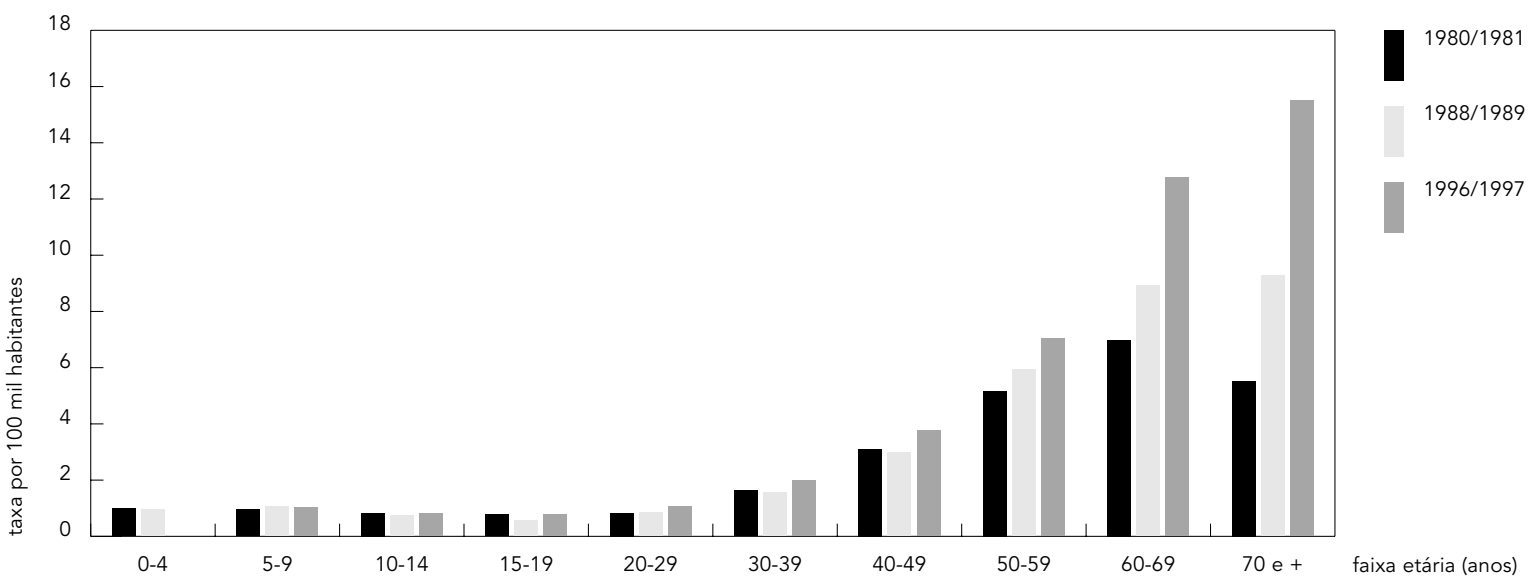

servou-se um aumento de $174 \%$ na mortalidade por tumores encefálicos em idosos, no período de 18 anos e, também aqui, o impacto foi maior entre as mulheres, com incremento claramente superior ao observado nos homens (Tabela 2).

Em relação às capitais selecionadas, no início do período estudado apenas Belém apresentava taxa padronizada inferior à do país, enquanto as demais apresentavam indicadores superiores a 3,0 óbitos por 100 mil habitantes, sendo a mais elevada em Goiânia (5,01/100 mil). Nos triênios seguintes, as taxas nessas cidades apresentaram oscilações importantes, ocorrendo certa equivalência ao final da série: quase todas apresentaram taxas entre 4 e 5 óbitos por 100 mil habitantes (Tabela 3). A maior elevação ocorreu em Belém (119,5\%), seguida por João Pessoa, São Paulo e Belo Horizonte, em contrapartida a Porto Alegre, onde ocorreu uma queda de $1,8 \%$, embora suas taxas nos demais triênios não apontassem tendência de redução.

No que tange à distribuição etária da mortalidade por tumores de encéfalo nessas capitais, repete-se o perfil de taxas reduzidas em menores de 30 anos, e crescentes nas faixas seguintes, principalmente nos maiores de 70 anos. Nesse aspecto, duas cidades se destacaram, João Pessoa e Belém. Comparando as taxas médias de mortalidade nos triênios 1980/ 1982 e 1995/1997, o extrato de 50 a 69 anos apresentou um aumento de $100,4 \%$ e os maiores de 70, 398,8\%, em João Pessoa. Em Belém, estes aumentos foram respectivamente de 325,4 e $824,3 \%$, do início ao final do período estudado (Tabela 3).

\section{Tumores das meninges}

A Tabela 4 apresenta a distribuição dos tumores de meninges segundo a localização anatômica de origem. Levando-se em conta as mudanças na classificação desses tumores da CID9 para a CID-10, analisou-se em separado a série de 1980 a 1995 (quadriênios) e a mortalidade do triênio 1996/1998.

No período de 1980 a 1995, observou-se que uma pequena parcela dos óbitos por tumores cerebrais teve origem nas meninges, variando entre $2,4 \%$ (50 em 2.098) e 2,7\% (113 em 4.191) do total desses tumores. Quanto à natureza tumoral, em média foram encontrados $27,1 \%$ de tumores malignos de meninges e $72,9 \%$ de benignos. Essas proporções apresentaram alguma variação durante o período, com os malignos chegando a corresponder a 44,8\% dos óbitos por meningiomas, em 1982.

As taxas de mortalidade por tumores das meninges cerebrais ajustadas por idade foram 0,06/100 mil, no primeiro quadriênio (1980/ 1983), e 0,08/100 mil, no último (1992/1995), correspondendo a uma elevação de $27,0 \%$ no período. As mulheres apresentaram taxas de mortalidade superiores às dos homens e, também, maior crescimento no período, observando-se que enquanto a taxa masculina aumentou $25,6 \%$, a feminina cresceu $47,0 \%$. Esse qua- 
dro se refletiu na razão de sexos, sendo da ordem de 0,6 nos primeiros quadriênios e 0,5 nos dois últimos.

As taxas de mortalidade por meningiomas foram crescentes com o aumento da idade. A redução observada nos menores de 30 anos deve ser considerada com cautela dado o número relativamente pequeno de óbitos. Comparando as taxas do quarto com o primeiro quadriênio, observou-se uma elevação relativamente homogênea nas faixas etárias subseqüentes, em ambos os sexos (Tabela 5).

No que tange às cidades selecionadas, as maiores taxas padronizadas, no início do período estudado (1980/1983), foram observadas em Belém e no Rio de Janeiro (0,20/100 mil em cada uma), enquanto que no último quadriênio (1992/1995) Brasília (0,29/100 mil) e São Paulo $(0,21 / 100 \mathrm{mil})$ apresentaram indicadores mais elevados. As taxas de mortalidade em cada cidade ao longo do período foram oscilantes em virtude do reduzido número de casos, não sendo possível observar tendências mais claras.

No período em que foi utilizada a CID-10 para a codificação dos óbitos (triênio 1996/1998), as neoplasias de meninges encefálicas benignas, malignas e de comportamento incerto ou desconhecido, representaram de $1,5 \%$ do conjunto de tumores de cérebro (211 em 13.623). Ao agregar os tumores de meninges sem especificação quanto ao sítio anatômico, passam a representar 3\% dos referidos tumores (423 em 13.623).

A taxa de mortalidade padronizada para o conjunto de tumores das meninges cerebrais e sem especificação anatômica foi de $0,11 / 100$ mil. Houve predominância de tumores benignos $(75,2 \%)$, tendo os de comportamento incerto ou desconhecido representado apenas $1,7 \%$ dos óbitos no período. O comportamento por sexo foi similar ao período anterior, com taxas padronizadas mais elevadas entre as mulheres $(0,14 / 100 \mathrm{mil})$ que entre os homens $(0,08 / 100$ mil).

\section{Discussão}

A taxa de mortalidade por tumores cerebrais, padronizada pela população mundial, mostrou um aumento de 49,9\% nos dezoito anos estudados, sendo as neoplasias encefálicas responsáveis por mais de $90 \%$ do total dos óbitos em todo o período. Essa predominância é esperada pelo pior prognóstico desses tumores em contraposição aos meningiomas, que são geralmente benignos.

\begin{tabular}{|c|c|c|c|c|c|}
\hline \multicolumn{6}{|c|}{$\begin{array}{l}\text { Distribuição de óbitos e taxas de mortalidade por tumores do encéfalo, } \\
\text { segundo sexo e faixa etária. Brasil, 1980/1981 e 1996/1997. }\end{array}$} \\
\hline \multirow{2}{*}{$\begin{array}{l}\text { Faixa etária } \\
\text { (anos) }\end{array}$} & \multicolumn{2}{|c|}{ 1980/1981 } & \multicolumn{2}{|c|}{$1996 / 1997$} & \multirow{2}{*}{$\begin{array}{c}\text { Variação (\%) } \\
\text { 1996/1997 } \\
\text { vs. 1980/1981 }\end{array}$} \\
\hline & $\mathrm{n}$ & Taxa* & $\mathrm{n}$ & Taxa* & \\
\hline \multicolumn{6}{|l|}{ Homens } \\
\hline$<15$ & 449 & 0,98 & 460 & 0,91 & $-6,9$ \\
\hline $15-29$ & 289 & 0,84 & 446 & 1,01 & 20,4 \\
\hline $30-49$ & 609 & 2,47 & 1.130 & 2,88 & 16,4 \\
\hline $50-69$ & 787 & 6,71 & 1.710 & 9,94 & 48,2 \\
\hline 70 e mais & 155 & 6,04 & 671 & 14,65 & 142,4 \\
\hline \multicolumn{6}{|l|}{ Mulheres } \\
\hline$<15$ & 367 & 0,81 & 399 & 0,81 & $-0,5$ \\
\hline $15-29$ & 270 & 0,76 & 322 & 0,72 & $-6,0$ \\
\hline $30-49$ & 462 & 1,83 & 903 & 2,19 & 19,1 \\
\hline $50-69$ & 541 & 4,45 & 1.376 & 7,28 & 63,5 \\
\hline 70 e mais & 133 & 4,30 & 780 & 13,40 & 211,5 \\
\hline \multicolumn{6}{|l|}{ Total } \\
\hline$<15$ & 817 & 0,90 & 859 & 0,86 & $-4,1$ \\
\hline $15-29$ & 559 & 0,80 & 768 & 0,86 & 7,7 \\
\hline $30-49$ & 1.071 & 2,15 & 2.034 & 2,52 & 17,4 \\
\hline $50-69$ & 1.328 & 5,56 & 3.087 & 8,54 & 53,6 \\
\hline 70 e mais & 288 & 5,09 & 1.453 & 13,95 & 174,0 \\
\hline
\end{tabular}

* Taxas por 100 mil habitantes.

A variabilidade na proporção de tumores malignos parece refletir, em primeiro lugar, a mudança no processo de codificação das declarações de óbito em virtude da introdução da décima revisão da CID, em 1996. A classificação anterior, CID-9, discriminava bem os tumores malignos e benignos do cérebro, mas quanto aos de natureza não especificada, apenas os encefálicos contavam com categoria própria (239.6: "neoplasma de natureza não especificada do encéfalo"), sendo os demais agrupados na subcategoria 239.7: "neoplasmas de natureza não especificada das glândulas endócrinas e de outras partes do sistema nervoso". No caso da declaração de óbito informar que a neoplasia apresentava comportamento incerto quanto à malignidade, os tumores encefálicos seriam classificados como 237.5: "neoplasma de comportamento incerto do encéfalo e da medula espinhal", e os das meninges (encefálicas, espinhais e sem localização especificada) como 237.6: "neoplasmas de comportamento incerto das meninges”. Assim, utilizando a CID-9, não se pode separar os óbitos por tumores de comportamento incerto com origem intracraniana, dos medulares. A CID-10 ampliou os có- 
Tabela 3

Distribuição de óbitos e taxas de mortalidade por tumores do encéfalo, segundo faixa etária em cidades selecionadas.

Brasil, 1980/1982 e 1995/1997.

\begin{tabular}{|c|c|c|c|c|c|c|c|c|}
\hline \multirow[t]{2}{*}{ Capital } & \multirow[t]{2}{*}{ Ano do óbito } & \multirow[t]{2}{*}{ Óbitos } & \multicolumn{5}{|c|}{ Faixa etária (anos) } & \multirow[t]{2}{*}{ Taxa padronizada* } \\
\hline & & & $<15$ & $15-29$ & $30-49$ & $50-69$ & 70 e + & \\
\hline \multirow[t]{2}{*}{ Belém } & $1980-1982$ & 46 & 1,17 & 0,97 & 2,71 & 2,60 & 1,79 & 1,85 \\
\hline & 1995-1997 & 116 & 0,95 & 1,18 & 4,10 & 11,04 & 16,58 & 4,06 \\
\hline \multirow[t]{2}{*}{ Belo Horizonte } & 1980-1982 & 145 & 1,64 & 1,01 & 2,80 & 10,14 & 10,74 & 3,53 \\
\hline & 1995-1997 & 261 & 2,56 & 1,08 & 3,07 & 13,52 & 27,88 & 4,14 \\
\hline \multirow[t]{2}{*}{ Curitiba } & 1980-1982 & 95 & 1,37 & 1,09 & 2,73 & 12,75 & 14,20 & 4,08 \\
\hline & 1995-1997 & 177 & 1,65 & 1,01 & 2,50 & 14,06 & 32,45 & 4,99 \\
\hline \multirow[t]{2}{*}{ Fortaleza } & 1980-1982 & 98 & 1,30 & 1,47 & 2,76 & 8,45 & 7,16 & 3,08 \\
\hline & 1995-1997 & 152 & 1,11 & 0,73 & 3,04 & 8,08 & 15,55 & 3,25 \\
\hline \multirow[t]{2}{*}{ Goiânia } & $1980-1982$ & 81 & 3,23 & 1,20 & 4,70 & 9,87 & 22,63 & 5,01 \\
\hline & $1995-1997$ & 111 & 1,26 & 1,46 & 2,95 & 13,02 & 34,30 & 5,17 \\
\hline \multirow[t]{2}{*}{ João Pessoa } & 1980-1982 & 25 & 1,62 & 0,94 & 3,80 & 6,79 & 3,76 & 3,01 \\
\hline & $1995-1997$ & 60 & 1,56 & 1,61 & 2,41 & 13,60 & 18,77 & 4,50 \\
\hline \multirow[t]{2}{*}{ Porto Alegre } & 1980-1982 & 137 & 1,84 & 1,71 & 3,65 & 9,96 & 20,00 & 4,34 \\
\hline & 1995-1997 & 168 & 1,01 & 0,91 & 3,11 & 11,81 & 25,38 & 4,26 \\
\hline \multirow[t]{2}{*}{ Rio de Janeiro } & 1980-1982 & 545 & 1,57 & 1,34 & 2,97 & 10,20 & 13,81 & 3,80 \\
\hline & 1995-1997 & 764 & 1,55 & 0,99 & 3,46 & 11,39 & 22,08 & 4,30 \\
\hline \multirow[t]{2}{*}{ São Paulo } & 1980-1982 & 693 & 1,47 & 0,82 & 3,02 & 8,78 & 10,08 & 3,29 \\
\hline & 1995-1997 & 1.192 & 1,30 & 1,12 & 3,10 & 12,16 & 27,04 & 4,54 \\
\hline
\end{tabular}

* Taxas por 100 mil habitantes padronizadas pela população mundial.

Tabela 4

Distribuição de freqüências de óbitos por neoplasias das meninges, segundo localização anatômica de origem.

Brasil, 1980-19981.

\begin{tabular}{|c|c|c|c|c|c|c|c|c|c|c|}
\hline \multirow{2}{*}{$\begin{array}{l}\text { Neoplasias } \\
\text { das meninges }\end{array}$} & \multicolumn{2}{|c|}{$1980 / 1983$} & \multicolumn{2}{|c|}{$1984 / 1987$} & \multicolumn{2}{|c|}{$1988 / 1991$} & \multicolumn{2}{|c|}{ 1992/1995 } & \multicolumn{2}{|c|}{$1996 / 1998$} \\
\hline & $\mathrm{n}$ & $\%$ & $\mathrm{n}$ & $\%$ & $\mathrm{n}$ & $\%$ & $\mathrm{n}$ & $\%$ & $\mathrm{n}$ & $\%$ \\
\hline Encefálicas 2 & 222 & 85,7 & 215 & 93,1 & 312 & 86,9 & 384 & 92,1 & 211 & 48,1 \\
\hline Espinhais 3 & 17 & 6,6 & 4 & 1,7 & 20 & 5,6 & 10 & 2,4 & 16 & 3,6 \\
\hline Outros 4 & 20 & 7,7 & 12 & 5,2 & 27 & 7,5 & 23 & 5,5 & & \\
\hline Meninges NE5 & & & & & & & & & 212 & 48,3 \\
\hline Total & 259 & 100,0 & 231 & 100,0 & 359 & 100,0 & 417 & 100,0 & 439 & 100,0 \\
\hline
\end{tabular}

1 Utilizou-se a CID-9 de 1980 a 1995 e a CID-10 em 1996/1898.

2 Neoplasias das meninges encefálicas: malignas (192.1 e C70.0), benignas (225.2 e D32.0) e

de comportamento incerto ou desconhecido (D42.0).

3 Neoplasias das meninges espinhais: malignas (192.3 e C70.1), benignas (225.4 e D32.1)

e de comportamento incerto ou desconhecido (D42.1).

4 Outros $=$ N. maligna de meninges que não se sabe sítio de origem (192.8) e de comportamento

incerto das glândulas endócrinas e outras partes do sistema nervoso (237.6), inclusive meninges.

5 Neoplasia das meninges com sítio não especificado: malignas (C70.9), benignas (D32.9)

e de comportamento incerto ou desconhecido (D42.9). 
digos permitindo maior especificação, mas essa mudança nos critérios de classificação dificulta a análise da série histórica dos meningiomas e neurinomas. Além disso, na CID-9, as regras de classificação orientavam considerar o óbito por "meningioma" (sem outra especificação) como benigno e encefálico, enquanto as regras da CID-10 orientam considerá-lo como benigno, sem localização informada. Assim, a aparente diminuição de meningiomas encefálicos deve-se a mudança na classificação, sendo agora codificados como neoplasias de meninges de localização anatômica não especificada (Tabela 4).

Em segundo lugar, vale destacar a variação no percentual de tumores encefálicos malignos e de natureza não especificada na vigência da CID-9, sugerindo que o processo de classificação desses tumores tampouco foi uniforme no período 1980/1995. No Brasil, tinha-se como norma de orientação aos codificadores das DOs, considerar como malignos os óbitos devidos às neoplasias, a menos que o próprio atestado informasse sua benignidade ou o comportamento incerto quanto à natureza tumoral. Tal orientação tinha por premissa que em nosso país as palavras "câncer", "neoplasia" e "tumor" têm uma conotação de malignidade tão intensa que os médicos usualmente não as utilizariam na DO se a doença que deu origem à morte não fosse maligna. Entretanto, essa assertiva não parecia pertinente para as neoplasias localizadas no cérebro, onde neoplasias benignas podem ser fatais pelo seu crescimento em local nobre, por ser centro de funções vitais e restrito pela calota craniana. Com isso, não se aplicava aquela orientação geral de supor malignidade, pois, na vigência da CID-9, os codificadores eram orientados para classificar como de "natureza não especificada" os óbitos devidos a neoplasias intracranianas que não trouxessem informação relativa à malignidade (Centro Brasileiro de Classificação de Doenças, 1996). Essa exceção foi eliminada na 10a Revisão que entrou em vigor no Brasil em 1996.

Assim, embora muitos estudos sobre os tumores encefálicos focalizem somente as neoplasias malignas, a observação dos dados de mortalidade do Brasil sugere fortemente a necessidade de se analisar de forma conjunta os óbitos classificados como de natureza maligna, benigna e não especificada.

A análise da mortalidade por tumores do encéfalo e das meninges cerebrais revela tanto conclusões similares como divergentes. Ambas apresentam incremento das taxas ao longo do período, notadamente nos maiores de 50 anos, sendo este aumento mais importante no sexo

\begin{tabular}{|c|c|c|c|c|c|}
\hline \multicolumn{6}{|c|}{$\begin{array}{l}\text { Distribuição de óbitos e taxas de mortalidade por tumores de meninges } \\
\text { encefálicas, segundo sexo e faixa etária. Brasil, 1980/1982 e 1992/1995. }\end{array}$} \\
\hline \multirow{2}{*}{$\begin{array}{l}\text { Faixa etária } \\
\text { (anos) }\end{array}$} & \multicolumn{2}{|c|}{$1980 / 1983$} & \multicolumn{2}{|c|}{$1992 / 1995$} & \multirow{2}{*}{$\begin{array}{c}\text { Variação (\%) } \\
\text { 1992/1995 } \\
\text { vs. 1980/1983 }\end{array}$} \\
\hline & $\mathrm{n}$ & Taxa* & $\mathrm{n}$ & Taxa* & \\
\hline \multicolumn{6}{|l|}{ Homens } \\
\hline$<15$ & 4 & 0,004 & 2 & 0,002 & $-56,2$ \\
\hline $15-29$ & 8 & 0,011 & 9 & 0,011 & $-6,7$ \\
\hline $30-49$ & 24 & 0,047 & 36 & 0,051 & 8,7 \\
\hline $50-69$ & 37 & 0,154 & 63 & 0,200 & 30,4 \\
\hline 70 e mais & 12 & 0,224 & 22 & 0,277 & 23,6 \\
\hline \multicolumn{6}{|l|}{ Mulheres } \\
\hline$<15$ & 2 & 0,002 & 3 & 0,003 & 32,1 \\
\hline $15-29$ & 8 & 0,011 & 9 & 0,010 & $-6,6$ \\
\hline $30-49$ & 38 & 0,073 & 71 & 0,097 & 32,7 \\
\hline $50-69$ & 65 & 0,259 & 121 & 0,352 & 36,2 \\
\hline 70 e mais & 24 & 0,369 & 48 & 0,479 & 29,7 \\
\hline \multicolumn{6}{|l|}{ Total } \\
\hline$<15$ & 6 & 0,003 & 5 & 0,002 & $-26,8$ \\
\hline $15-29$ & 16 & 0,011 & 18 & 0,011 & $-6,7$ \\
\hline $30-49$ & 62 & 0,060 & 107 & 0,074 & 23,8 \\
\hline $50-69$ & 102 & 0,207 & 184 & 0,280 & 35,0 \\
\hline 70 e mais & 36 & 0,303 & 70 & 0,389 & 28,3 \\
\hline
\end{tabular}

* Taxas por 100 mil habitantes.

feminino. Por outro lado, o crescimento das taxas de mortalidade por tumores encefálicos é de magnitude bastante superior ao dos meningiomas $(44,7 \%$ versus $27,0 \%)$. Além disso, enquanto os percentuais de aumento dos tumores de meninges são similares nos extratos de 50 a 69 e maiores de 70 anos (Tabela 5), nos encefálicos o grupo maior de 70 anos apresenta crescimento muito superior ao de 50 a 69 (Tabela 2). Essas observações sugerem que o aumento das taxas de mortalidade por tumores do encéfalo não deve ser creditado apenas à disponibilidade de tecnologia diagnóstica que é utilizada tanto para estes tumores quanto para os meningiomas, embora não se possa descartar que tenha ocorrido uma elevação na incidência destes, não acompanhada pela mortalidade.

Ainda em relação aos meningiomas, é importante ponderar que as variações das taxas podem ser devidas a flutuações aleatórias, dado o pequeno número de óbitos, especialmente entre os menores de 30 anos.

Quando se considera a variação das taxas de mortalidade por tumores encefálicos nas ci- 
dades selecionadas, ficam evidentes os diferenciais nos níveis de incremento que foram bem mais elevados nas capitais do Norte e Nordeste do que nas do Sul e Sudeste. Este comportamento merece ser melhor estudado para avaliar se a magnitude da disponibilidade e acesso a especialistas e meios radiológicos para diagnóstico de tumores cerebrais ocorreu em épocas diferentes nessas áreas. Além disso, também merece ser investigada a introdução e a distribuição de potenciais fatores de risco nas cidades analisadas.

A mortalidade por câncer de cérebro, nos Estados Unidos, tem sido estudada por vários autores. Garfinkel \& Sarokhan (1982) analisaram o período de 1940 a 1977, Modan et al. (1992) estudaram o período de 1968 a 1988, e Legler et al. (1999) analisaram a mortalidade de 1975 a 1995. No primeiro período, Garfinkel \& Sarokhan (1982) constataram um crescimento lento das taxas de mortalidade por câncer de cérebro. A distribuição por faixa etária já apresentava o comportamento clássico de um padrão semelhante em ambos os sexos, caracterizado por taxas reduzidas na infância, menores nos adolescentes, maiores nos adultos jovens, aumentando de forma importante nos mais velhos e atingindo o pico na faixa de 70 a 74 anos, com subseqüente decréscimo após os 75 anos.

No período de 1968 a 1988, Modan et al. (1992) observaram um crescimento gradual e consistente na taxa de mortalidade por câncer de cérebro nos maiores de 64 anos, mas não entre os mais jovens. Comparando o primeiro e o último ano, constataram um aumento de $50 \%$ na faixa etária de 65 a 74 anos, de $200 \%$ na de 75 a 84 anos e de $800 \%$ nos maiores de 85 , sendo este padrão similar em ambos os sexos. Analisando a conduta diagnóstica e terapêutica, no período analisado, observaram um aumento importante na utilização de procedimentos diagnósticos, mas não nas cirurgias. Com isso, concluíram que o aumento observado seria resultado da disponibilidade de tecnologia diagnóstica mais precisa e menos invasiva, acessível a idosos.

Essa conclusão foi corroborada pelo estudo de Legler et al. (1999), que constataram uma queda na mortalidade por câncer de cérebro em menores de 45 anos, nos Estados Unidos, de 1975 a 1995. A taxa do grupo de 45 a 64 anos foi relativamente constante, enquanto a dos maiores de 64 anos mostrou-se crescente até 1990, tornando-se estável depois desse ano. Uma análise mais detalhada desse último grupo detectou um aumento de $0,9 \%$ ao ano na faixa etária de 65 a 74 anos, enquanto a de 75 a 84 apresentou crescimento no início do perío- do, seguido de estabilização entre 1978 e 1989, sendo constatado um decréscimo de $0,04 \%$ ao ano, sem significância estatística, entre 1990 e 1995. Por outro lado, as taxas de mortalidade dos maiores de 84 anos registraram um aumento anual de $8,5 \%$ até 1988 , e de $2,1 \%$ ao ano de 1988 a 1995. Os autores chamam a atenção para o nivelamento das taxas de incidência e de mortalidade dessas três faixas etárias no ano de 1995.

Davis et al. (1990) analisaram a mortalidade por tumores cerebrais em cinco países desenvolvidos, comparando as taxas de $1968 \mathrm{com}$ as de 1986/1987. Observaram um aumento substancial nas taxas dos maiores de 65 anos e um excesso de mortes entre os homens, em todos os países. Esse aumento de taxas foi similar em homens e mulheres na Alemanha, Estados Unidos e Reino Unido, sendo porém maior entre as mulheres na Itália e na França. Os autores constataram, ainda, que esse incremento teve início antes de se tornarem disponíveis recursos diagnósticos mais refinados como a tomografia computadorizada e a ressonância magnética para aquelas populações. Além disso, as taxas de mortalidade mantiveram a tendência crescente nos anos seguintes à introdução dessas técnicas, quando se esperaria sua estabilização, uma vez que não se registraram mudanças importantes no acesso a esses meios diagnósticos.

Mao et al. (1991) analisaram o aumento da mortalidade por câncer de cérebro no Canadá no período de 1959 a 1988, usando taxas anuais padronizadas pela população do país em 1971. Estratificando por sexo e idade (menores de 65 anos; 65 e mais anos), observaram uma variação anual crescente e reduzida nos menores de 65 anos $(0,3 \%$ nos homens e $0,1 \%$ nas mulheres) e maior naqueles com 65 e mais anos $(4,1 \%$ nos homens e $4,5 \%$ nas mulheres). A análise por coorte de nascimento (qüinqüênios), dos que morreram com 40 anos ou mais, não identificou a presença de efeito coorte.

Shugg et al. (1994) compararam as taxas de mortalidade por tumores de cérebro, padronizadas pela população mundial, de dois registros de câncer de base populacional da Austrália. As taxas médias de mortalidade dos dois períodos analisados (1978/1985 e 1996/1992) foram semelhantes: 5,3 e 5,2/ 100 mil para o sexo masculino, e 3,3 e 3,4/ 100 mil para o sexo feminino, respectivamente. Observaram um aumento importante tanto nos menores de 15 anos (incremento de $25,0 \%$ no sexo feminino e $44,4 \%$ no masculino), quanto nos maiores de 75 anos $(51,5 \%$ nas mulheres e $80,9 \%$ nos homens). 
Em consonância com a literatura, no presente trabalho observou-se uma variação das taxas de mortalidade por tumores de cérebro de acordo com a idade, apresentando um incremento maior entre os mais idosos (Davis et al., 1990; Garfinkel \& Sarokhan, 1982; Legler et al., 1999; Mao et al., 1991; Modan et al., 1992; Shugg et al., 1994; Werner et al., 1995). Também o predomínio masculino nos óbitos por tumores encefálicos (razão de sexos de 1,3) e o feminino nos meningiomas (razão de sexos de 0,5 ) foram semelhantes ao descrito na literatura (Fleury et al., 1997; Gold, 1982; Shugg et al., 1994). A observação de maior magnitude do aumento da mortalidade por tumores cerebrais entre as mulheres no Brasil concorda com o estudo de Davis et al. (1990), em relação ao comportamento daquelas taxas na Itália e na França.

As taxas de mortalidade brasileiras aqui apresentadas são muito menores do que as reportadas por Shugg et al. (1994) nos registros de base populacional da Austrália. O crescimento das taxas australianas foi menos pronunciado nos idosos, especialmente entre as mulheres.

As taxas brutas de mortalidade por tumores cerebrais apresentaram um crescimento constante no período de 18 anos, variando de 1,78 para 2,83/100 mil. Essa evolução poderia ser explicada pelo envelhecimento populacional, uma vez que esses tumores apresentam taxas muito mais elevadas entre os mais idosos. Entretanto, essa explicação responde apenas por parte do aumento, uma vez que, mesmo após a padronização por idade, ele foi da ordem de $49,9 \%$ (de 2,24 para $3,35 / 100$ mil).

Esse incremento poderia ser parcialmente devido à melhora do SIM, no período estudado. Sabe-se que a mortalidade proporcional por causas mal definidas de óbito diminuiu de 22 para $15 \%$ no Brasil, entre 1980 e 1998. Entretanto, as localizações mal definidas dentro do capítulo das neoplasias apresentaram uma variação bem menor, 0,06 para $0,02 \%$ no mesmo período. Assim, parece que a melhoria do sistema de mortalidade seria suficiente por si para explicar apenas parte do aumento observado na notificação de mortes por tumores cerebrais no país.

O total de exames pagos pelo Sistema Único de Saúde (SUS) pode ser obtido por meio do DATASUS (Departamento de Informática do SUS), que disponibiliza o quantitativo dos diversos procedimentos a partir de julho de 1994, via Internet (http://www.datasus.gov.br). Em 1995, foram apresentados ao SUS um total de 463.590 tomografias computadorizadas de crâ- nio e coluna (29,5 exames por 10 mil habitantes), e em 1999, 632.608 (38,6/10 mil habitantes), perfazendo um aumento de $30,7 \%$ deste indicador para o país no referido período. As cidades que tiveram maior crescimento na taxa de mortalidade por tumores encefálicos entre 1980/1982 e 1995/1997, apresentaram comportamentos distintos na variação nos coeficientes populacionais de tomografias computadorizadas realizadas em 1995 e em 1999. Enquanto Belém mostrou um incremento expressivo de 10,6/ 10 mil, em 1995, para 48,4/ 10 mil, em 1999 (357,7\%), João Pessoa apresentou declínio de 37,6\% (de 93,4/ 10 mil para 58,3/10 mil) no mesmo intervalo de tempo. Em Porto Alegre, houve uma queda de 1,8\% nas taxas de mortalidade de 1980/1982 a 1995/1997 e um aumento de $9,8 \%$ nos coeficientes de tomografias computadorizadas de crânio e coluna realizadas nos anos de 1995 e 1999.

Considerando as evidências aqui descritas, de que a ampliação de acesso aos meios diagnósticos e o envelhecimento populacional poderiam explicar apenas parcialmente o incremento observado na mortalidade por tumores cerebrais, faz-se necessário estudar o comportamento de fatores de risco em potencial para estas neoplasias na realidade brasileira. A literatura destaca a exposição a produtos químicos, notadamente pesticidas e solventes, em ambiente domiciliar e, principalmente, ocupacional (Bohnen \& Kurland, 1995; Inskip et al., 1995; Preston-Martin, 1996; Preston-Martin et al., 1989; Thomas \& Waxweiler, 1986). A utilização de pesticidas está documentada pela variação em sua venda no território nacional, mostrando importante aumento ao longo dos anos (Meyer et al., 1999). A associação entre a incidência de tumores cerebrais e exposição ocupacional a solventes e outros produtos químicos em nosso meio merece ser investigada, assim como a exposição à radiação eletromagnética de baixa freqüência $(60 \mathrm{~Hz})$ originada pela proximidade às linhas de alta tensão elétrica, estações e subestações de eletricidade, pois suspeita-se que esta última possa atuar como um fator de promoção tumoral para câncer de cérebro (Brem et al., 1995; Inskip et al., 1995; Preston-Martin, 1996; Preston-Martin et al., 1989; Ryan et al., 1992).

Outra fonte de exposição a produtos químicos seria o emprego de cosméticos, especialmente entre mulheres. Também nesse campo houve um crescimento considerável tanto na diversificação de produtos quanto em sua utilização nas últimas décadas. Entre tais produtos, o contato com tinturas de cabelo, tanto no manejo profissional como no uso pessoal, tem 
sido relacionado a neoplasias de diferentes localizações, como cérebro, mama, ovário e vesícula (Inskip et al., 1995; Preston-Martin, 1996; Teta et al., 1984; Thomas \& Waxweiler, 1986). Em estudo de fatores de risco para neoplasias malignas de cérebro em crianças realizado no Rio de Janeiro, Koifman et al. (1998) encontraram um risco aproximadamente três vezes maior em mães de casos que em mães de controles quanto ao emprego de tinturas e alisantes de cabelos durante a gestação.

A observação de maior aumento dos tumores encefálicos entre as mulheres levanta questionamentos quanto a possíveis explicações, demandando pesquisas que levem em conta o longo período de latência entre exposição e desenvolvimento da neoplasia. A inserção em ambientes laborais, antes restritos aos homens, poderia ser interpretada como um elemento favorável à hipótese dos fatores de risco ocupacionais. Também o uso de cosméticos, especialmente tinturas de cabelo, merece investigação e acompanhamento de grupos expostos ao longo do tempo.

\section{Agradecimentos}

Os autores agradecem a Rosalina Jorge Koifman, Inês Echenique Mattos e Rosângela Alves Pereira por suas sugestões e comentários críticos. Agradecem, também, a Angela Cascão pela assessoria nas revisões da Classificação Internacional de Doenças.

\section{Conclusão}

A taxa de mortalidade por tumores cerebrais no Brasil, no período de 1980 a 1998, apresentou aumento, especialmente nos idosos e nas mulheres. A taxa padronizada pela população mundial, cresceu de 2,24/100 mil (1980) para 3,35/100 mil (1998). Este crescimento parece ser explicado apenas parcialmente pela maior disponibilidade de acesso aos cuidados com a saúde, em particular aos meios de diagnóstico por imagem. A elucidação dos fatores relacionados com esse incremento permitiria a elaboração de práticas preventivas com vistas à redução desses tumores que, embora de baixa magnitude, são de grande importância devido à alta letalidade.

Novos estudos devem ser desenvolvidos, tanto para dimensionar com melhor precisão a contribuição das novas tecnologias diagnósticas introduzidas nos últimos 30 anos, quanto para estimar a associação da exposição a potenciais fatores de risco para esses tumores na modificação de seu padrão de distribuição observado no Brasil.

\section{Referências}

AHLBOM, A., 1990. Some notes on brain tumor epidemiology. Annals of New York Academy of Sciences, 609:179-185.

BAHEMUKA, M.; MASSEY, E. W. \& SCHOENBERG, B. S., 1988. International mortality from primary nervous system neoplasms: Distribution and trends. International Journal of Epidemiology, 17:33-38.

BOHNEN, N. I. \& KURLAND, L. T., 1995. Brain tumor and exposure to pesticides in humans: A review of the epidemiologic data. Journal of the Neurological Sciences, 132:110-121.

BREM, S.; ROZENTAL, J. M. \& MOSKAL, J. R., 1995. What is the etiology of human brain tumors? Cancer, 76:709-713.

CENTRO BRASILEIRO DE CLASSIFICAÇÃO DE DOENÇAS, 1996. Codificação de neoplasias e tumores. Boletim do Centro da OMS para a Classificação de Doenças em Português, 17:6.

COLEMAN, M. P.; ESTEVE, J.; DAMIECKI, P.; ARSLAN, A. \& RENARD, H., 1993. Material. Trends in Cancer Incidence and Mortality. IARC Scientific Publications 121. Lyon: International Agency for Research on Cancer.

DATASUS (Departamento de Informática do SUS), 2000. Sistema de Informação sobre Mortalida- 
de 1979-1998: Dados de Declaração de Óbito. CDROM. Brasília: Ministério da Saúde.

DAVIS, D. L.; HOEL, D.; PERCY, C.; AHLBOM, A. \& SCHWARTZ, J., 1990. Is brain cancer mortality increasing in industrial countries? Annals of the New York Academy of Sciences, 609:191-204.

DESMEULES, M.; MIKKELSEN, T. \& MAO, Y., 1992. Increasing incidence of primary malignant brain tumors: Influence of diagnostic methods. Journal of the National Cancer Institute, 84:442-445.

FLEURY, A.; MENEGOZ, F; GROSCLAUDE, P; DAURES, J. P.; HENRY-AMAR, M.; RAVERDY, N.; SCHAFFER, P.; POISSON, M. \& CELATTRE, J. Y., 1997. Descriptive epidemiology of cerebral gliomas in France. Cancer, 79:1195-1202.

GARFINKEL, L. \& SAROKHAN, B., 1982. Trends in brain cancer tumor mortality and morbidity in the United States. Annals of New York Academy of Sciences, 381:1-5.

GOLD, E. B., 1982. Epidemiology of brain tumors. In: Reviews in Cancer Epidemiology (A. M. Lillienfeld, ed.), v. 1, pp. 245-292, New York: Elservier/Amsterdam: North-Holland.

GREENWALD, P.; FRIEDLANDER, B. R.; LAWRENCE, C. E.; HEARNE, T. \& EARLE, K., 1981. Diagnostic sensitivity bias - An epidemiologic explanation for apparent brain tumor excess. Journal of Occupational Medicine, 23:690-694.

GREIG, N. H.; RIES, L. G.; YANCIK, R. \& RAPOPORT, S. I., 1990. Increasing annual incidence of primary malignant brain tumor in the elderly. Journal of the National Cancer Institute, 82:1621-1624.

HELSETH, A., 1995. The incidence of primary central nervous system neoplasms before and after computerized tomography availability. Journal of Neurosurgery, 83:999-1003.

INSKIP, P. D.; LINET, M. S. \& HEINEMAN, E. F., 1995. Etiology of brain tumors in adults. Epidemiologic Reviews, 17:382-414.

KOIFMAN, S., 1995. Incidência de câncer no Brasil. In: Os Muitos Brasis - Saúde e População na Década de 80 (M. C. S. Minayo, org.), pp. 143-176, São Paulo: Editora Hucitec/Rio de Janeiro: ABRASCO.

KOIFMAN, S.; KOIFMAN, R. J. \& LEITÃO, C. R., 1998. Environmental risk factors associated to brain cancer during childhood in Rio de Janeiro, Brazil: Preliminary results. In: 17 th International Cancer Congress, Abstract Book, p. 287, Rio de Janeiro: Instituto Nacional do Câncer/Union Internationale Contre le Cancer.

LEGLER, J. M.; RIES, L. A. G.; SMITH, M. A.; WARREN, J. L.; HEINEMAN, E. F.; KAPLAN, R. S. \& LINET, M. S., 1999. Brain and other central nervous system cancers: Recent trends in incidence and mortality. Journal of the National Cancer Institute, 91: 1382-1390.

MAO, Y. M.; DESMEULES, M.; SEMENCIW, R. M.; HILL, G.; GAUDETTE, L. \& WIGLE, D. T., 1991. Increasing brain cancer rates in Canada. Canadian Medical Association Journal, 145:1583-1591.

MEYER, A.; SARCINELLI, P. N. \& MOREIRA, J. C., 1999. Estarão alguns grupos populacionais brasileiros sujeitos à ação de disjuntores endócrinos? Cadernos de Saúde Pública, 15:845-850.

MODAN, B.; WAGENER, D. K.; FELDMAN, J. J.; ROSENBERG, H. M. \& FEINLEIB, M., 1992. Increased mortality from brain tumors: A combined outcome of diagnostic technology and change of attitude toward the elderly. American Journal of Epidemiology, 135:1349-1357.

MONTEIRO, G. T. R.; KOIFMAN, R. J. \& KOIFMAN, S., 1997. Confiabilidade e validade dos atestados de óbito por neoplasias. I. Confiabilidade da codificação para o conjunto das neoplasias no Estado do Rio de Janeiro. Cadernos de Saúde Pública, 13 (Sup. 1):39-52.

OMS (Organização Mundial da Saúde), 1985. Manual da Classificação Estatística Internacional de Doenças, Lesões e Causas de Óbito. 9a Revisão. v. 1. São Paulo: Centro Brasileiro de Classificação de Doença.

OMS (Organização Mundial da Saúde), 1997. Classificação Estatística Internacional de Doenças e Problemas Relacionados à Saúde. 10a Revisão. v. 1. São Paulo: Centro Brasileiro de Classificação de Doença/Editora Universidade de São Paulo.

PAULA, A. M. C.; EVANGELISTA FILHO, D.; PEREIRA, I. P. A.; ALBANO, A. H. B. L. \& FERNANDES, R. M., 1994. Avaliação dos dados de mortalidade, Brasil - 1979 a 1989. Informe Epidemiológico do SUS, 3:21-41.

PRESTON-MARTIN, S., 1996. Epidemiology of primary CNS neoplasms. Neuroepidemiology, 14:273290.

PRESTON-MARTIN, S.; MACK, W. \& HENDERSON, B. E., 1989. Risk factors for gliomas and meningiomas in males in Los Angeles County. Cancer Research, 49:6137-6143.

RYAN, P.; LEE, M. L.; NORTH, J. B. \& McMICHAEL, A. J., 1992. Risk factors for tumors of the brain and meninges: Results from the Adelaide Adult Brain Study. International Journal of Cancer, 51:20-27.

SEGI, M., 1960. Cancer Mortality for Selected Sites in 24 Countries (1950-57). Sendai: Department of Public Health, Tohoku University School of Medicine.

SHUGG, D.; ALLEN, B. J.; BLIZZARD, L.; DWYER, T. \& RODES, D., 1994. Brain cancer incidence, mortality and case survival: Observations from two Australian cancer registries. International Journal of Cancer, 59:765-770.

SIQUEIRA, A. A. F.; RIO, E. M. B.; TANAKA, A. C. A.; SCHOR, N.; ALVARENGA, A. T. \& ALMEIDA, L. C., 1999. Mortalidade feminina na região sul do $\mathrm{Mu}$ nicípio de São Paulo: Qualidade da certificação médica dos óbitos. Revista de Saúde Pública, 33: 499-504.

TETA, M. J.; WALRATH, J.; MEIGS, J. W. \& FLANNERY, J. T., 1984. Cancer incidence among cosmetologists. Journal of the National Cancer Institute, 72: 1051-1057.

THOMAS, T. L. \& WAXWEILER, R. J., 1986. Brain tumors and occupational risk factors. Scandinavian Journal of Working Environmental Health, 12:115.

WERNER, M. H.; PHUPHANICH, S. \& LYMAN, G. H., 1995. The increasing incidence of malignant gliomas and primary central nervous system lymphoma in the elderly. Cancer, 78:1634-1642.

Recebido em 15 de agosto de 2002

Versão final reapresentada em 7 de janeiro de 2003

Aprovado em 22 de abril de 2003 